\title{
Parieto-Frontal Connectivity during Visually Guided Grasping
}

\author{
Meike J. Grol, ${ }^{1,2,5}$ Jasminka Majdandžić, ${ }^{2,3}$ Klaas E. Stephan, ${ }^{2,4}$ Lennart Verhagen, ${ }^{1,2}$ H. Chris Dijkerman, \\ Harold Bekkering, ${ }^{2,3}$ Frans A. J. Verstraten, ${ }^{1}$ and Ivan Toni ${ }^{2,3}$ \\ ${ }^{1}$ Helmholtz Institute, Experimental Psychology, Universiteit Utrecht, 3508 TC Utrecht, The Netherlands, ${ }^{2}$ F. C. Donders Centre for Cognitive Neuroimaging \\ and ${ }^{3}$ Nijmegen Institute for Cognition and Information, Radboud University Nijmegen, NL-6500 Nijmegen, The Netherlands, ${ }^{4}$ Wellcome Trust Centre for \\ Neuroimaging, University College London, London WC1N 3BG, United Kingdom, and ${ }^{5}$ Leiden Institute for Brain and Cognition, Leiden University, \\ 2300 RC, Leiden, The Netherlands
}

Grasping an object requires processing visuospatial information about the extrinsic features (spatial location) and intrinsic features (size, shape, orientation) of the object. Accordingly, manual prehension has been subdivided into a reach component, guiding the hand toward the object on the basis of its extrinsic features, and a grasp component, preshaping the fingers around the center of mass of the object on the basis of its intrinsic features. In neural terms, this distinction has been linked to a dedicated dorsomedial "reaching" circuit and a dorsolateral "grasping" circuit that process extrinsic and intrinsic features, linking occipital areas via parietal regions with the dorsal and ventral premotor cortex, respectively. We have tested an alternative possibility, namely that the relative contribution of the two circuits is related to the degree of on-line control required by the prehension movement.

We used dynamic causal modeling of functional magnetic resonance imaging time series to assess how parieto-frontal connectivity is modulated by planning and executing prehension movements toward objects of different size and width. This experimental manipulation evoked different movements, with different planning and execution phases for the different objects. Crucially, grasping large objects increased inter-regional couplings within the dorsomedial circuit, whereas grasping small objects increased the effective connectivity of a mainly dorsolateral circuit, with a degree of overlap between these circuits. These results argue against the presence of dedicated cerebral circuits for reaching and grasping, suggesting that the contributions of the dorsolateral and the dorsomedial circuits are a function of the degree of on-line control required by the movement.

Key words: reaching; manual prehension; V3A; V6A; anterior intraparietal area; premotor cortex; on-line control

\section{Introduction}

It has been suggested that, when we grasp an object, the brain needs to extract visuospatial information about the spatial location of the object relative to the subject (extrinsic features), as well as information about its size, shape, and orientation (intrinsic features) (Arbib, 1981). Kinematic data show that varying object size affects the maximum hand aperture, whereas varying object distance affects the kinematic profile of the reaching limb (Jeannerod, 1984). These findings have led to the suggestion that manual prehension is controlled through two visuomotor channels: a reach component, transporting the hand toward the object, and a grasp component, preshaping the fingers according to the size

\footnotetext{
Received April 24, 2007; accepted Sept. 13, 2007.

This work was supported by The Netherlands Organization for Scientific Research (NW0) Pioneer Grant (M.J.G., F.A.J.V.) and NWO Vidi Grant 452-03-339 (I.T.). We thank Paul Gaalman for excellent assistance during MR scanning, the Intention and Action group for invaluable discussions and comments, and Jan Matthijs Schoffelen for excellent help on the illustrations. The authors' contributions were as follows: experimental design (J.M., H.B., I.T.); data collection (J.M., M.J.G., L.V.); data analysis (M.J.G., J.M., K.E.S., I.T., L.V.); and manuscript preparation (M.J.G., J.M., K.E.S., I.T., H.C.D., L.V., F.A.J.V., H.B.).

Correspondence should be addressed to Dr. Meike J. Grol, Helmholtz Institute, University of Utrecht, P.O. Box 80125, 3508 TC Utrecht, E-mail: M.J.Grol@lumc.nl; or F. C. Donders Centre for Cognitive Neuroimaging, Radboud University Nijmegen, NL-6500 HB Nijmegen, The Netherlands.

D01:10.1523/JNEUROSCI.3923-07.2007

Copyright $\odot 2007$ Society for Neuroscience $\quad$ 0270-6474/07/2711877-11\$15.00/0
}

and the center of mass of the object (Jeannerod, 1988). This functional organization appears to have a physiological counterpart in two anatomically segregated parieto-frontal circuits: a dorsolateral circuit, consisting of an anterior intraparietal (AIP) area connected to the rostral part of the ventral premotor cortex (PMv; area F5), and a dorsomedial circuit, consisting of the anterior portion of the occipito-parietal sulcus (area V6A) and the caudal dorsal premotor cortex (PMd; area F2) (Tanne-Gariepy et al., 2002; Galletti et al., 2003). The dorsolateral circuit has been linked to the grasping component of prehension (Jeannerod et al., 1995). AIP contains neurons that are selectively activated during specific grasping movements and respond to threedimensional shape, size, and orientation of objects, while remaining insensitive to the position of an object relative to the animal (Murata et al., 2000). Disruption of AIP leads to severe impairments in the preshaping of the hand during grasping (Gallese et al., 1994; Tunik et al., 2005). Area F5 is crucially involved in planning and executing grasping movements (Fogassi et al., 2001; Davare et al., 2006). In contrast, the dorsomedial circuit has been linked to the reaching component (Burnod et al., 1999). Area V6A in macaques contains reaching cells (Fattori et al., 2001, 2005) and visuomotor neurons coding object position in space (Galletti et al., 1999). Disruption of V6A leads to errors in 
reaching (Battaglini et al., 2002; Karnath and Perenin, 2005), and area F2 is important for planning arm movements (Wise et al., 1997).

These findings could suggest a functional dichotomy between reaching and grasping organized along dorsolateral and dorsomedial pathways; yet, no double dissociation between reaching and grasping deficits in neuropsychological studies has been found (Pisella et al., 2006). Accordingly, some authors have proposed more integrated control mechanisms of prehension (Haggard and Wing, 1995; Zaal et al., 1998; Smeets and Brenner, 1999; Ulloa and Bullock, 2003), but it remains unclear how to implement these mechanisms within the segregated dorsomedial and dorsolateral parieto-frontal circuits.

Here, we explore a neglected feature of the cerebral activity supporting reachinggrasping movements, both in human and macaque studies. We used dynamic causal modeling (DCM) (Friston et al., 2003) on functional magnetic resonance imaging (fMRI) time series acquired during planning and execution of visually guided reaching-to-grasp movements toward objects of different size to explore the interregional couplings between regions of the dorsolateral (AIP and PMv) and the dorsomedial (V6A and PMd) circuits. By assessing how different hand-object interactions modulate the effective connectivity within this network, we tested the hypothesis that the involvement of the dorsolateral and dorsomedial parieto-frontal circuits is related to the degree of on-line control required by the prehension movement.

\section{Materials and Methods}

\section{Subjects}

Twenty healthy right-handed male volunteers (25 \pm 4 years) were recruited to participate in the study. They all had normal or corrected-tonormal vision and gave informed consent according to institutional guidelines of the local ethics committee (Commissie Mensgebonden Onderzoek region Arnhem-Nijmegen, Netherlands). Data from three subjects were discarded because of head-movement artifacts during the magnetic resonance (MR) scanning.

Six additional right-handed volunteers were recruited to participate in a behavioral control experiment, to measure kinematics parameters of the reaching-to-grasp movements and to compare these with the kinematics of reaching-to-point movements (see supplemental material, available at www.jneurosci.org).

\section{Experimental setup}

Subjects had to perform reaching-to-grasp and place movements while lying supine in the MR scanner. The standard mattress of the scanner bed was removed, allowing the subjects to lie considerably lower within the bore of the scanner to enable them to comfortably bend their head. Their head was fitted inside a phased-array receiver head coil. The head coil was tilted forward by $30^{\circ}$ along the subject's sagittal plane (Fig. $1 \mathrm{~A}$ ). This setup allowed the subjects to have a direct line of sight of the objects to be grasped and to visually control their movements. The subjects were not

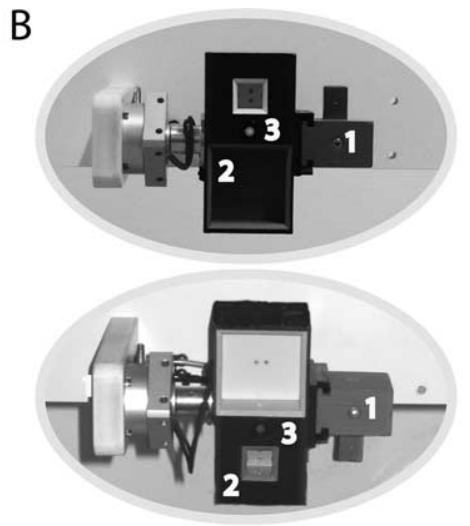

LARGE

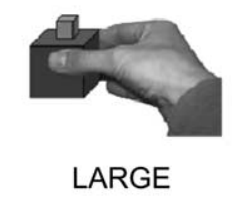

$\square$

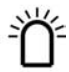

Figure 1. Experimental design. $\boldsymbol{A}$, Experimental setup. Subjects had to perform visually guided grasping movements and object manipulations while lying supine in the MRscanner. Their head was fitted inside a phased-array receiver head coil. The head 列 served as a home key on which subjects had to keep their hand in between trials. This device recorded subjects' reaction and movement times. $\boldsymbol{B}$, Grasping device. The subjects were instructed to grasp and manipulate an object (1) consisting of a large red cube and a small green cube, attached to a supporting rail positioned in front of them. The object (1) was positioned next to a rectangular box (2) containing two cubic slots. The object was held in place through an arc-shaped device positioned over the , Experimental time course. During the task, in each trial, subjects had to grasp the object either at the large (LARGE) or the smal part, remove the object from the rail to put it into one of the two slots, and reposition the object in the supporting rail. variable amount of time $(2-7 \mathrm{~s})$, so that the subject could not predict cue onset. The gray areas refer to the movement planning (RT) and movement execution (MT) phases considered in the DCM.

asked to fixate their eyes, so that they could freely explore the visual scene. An optical response button box (MRI Devices, Waukesha, WI), positioned on the upper leg (Fig. 1A, number 4), served as a home key on which subjects had to keep their hand in between trials. This device allowed us to record subjects' reaction times (RTs) and total movement times.

We ensured that subjects performed the task by moving their right forearm only. This was achieved by fitting a plastic splint around the elbow and by firmly, but comfortably, strapping the arm to the scanner table. The splint constrained the rotations around the elbow to the plane between the home key and the target object, minimizing the movements around the shoulder. The subject's head was kept in place using foam wedges.

The subjects were instructed to grasp and manipulate an object consisting of a large red cube and a small green cube, attached to a supporting rail positioned in front of them. The object was held in place through an arc-shaped device positioned over the subject's hips inside the MR scanner (Fig. 1A). The object (Fig. $1 B$, number 1 ) was positioned next to a rectangular box (Fig. $1 B$, number 2 ) containing two cubic slots. The subject could comfortably perform visually guided reaching-grasping 
movements toward the object, extract the object from the supporting rail, insert the object into one of the slots, and finally reposition the object in the supporting rail. The subjects had to insert the object back into the rail to ensure that the starting position of the larger cube did not vary over trials. Whether the small or large object was on top could be varied by means of a computer-controlled pneumatic mechanism.

A light-emitting diode (LED) was installed in the middle of each of the two sides of the box (Fig. $1 B$, number 3 ). The color of the LED instructed the subject on the movement to perform. MR-compatible switches located at various positions on the device recorded the time at which the object was removed from the supporting rail, the time at which the object was inserted into one of the slots, and the time at which the object was put back into the supporting rail. Control of the pneumatic rotation mechanism and recording of the movement-related responses was performed using a personal computer running Presentation 0.81 (Neurobehavioral Systems, San Francisco, CA).

\section{Experimental time course and procedures}

During the task, in each trial subjects had to grasp the object at either the large (LARGE) or the small (SMALL) part (Fig. 1C), remove the object from the rail to put it into one of the two slots, and finally reposition the object in the supporting rail. When the LED switched on, subjects had to leave the home key as soon as possible, make the appropriate object manipulation, and return to the home key. After $6 \mathrm{~s}$, the LED switched off, and a baseline of variable length (1.5-6 s) followed. Subjects were instructed to complete their action before the LED switched off. At the beginning of each block, the box was rotated, followed by a variable amount of time (2-7 s), so that the subject could not predict cue onset. The time course of a trial is shown in Figure $1 D$. Each block contained a randomized number of three to nine trials. Subjects first had a $15 \mathrm{~min}$ training session outside the scanner, until error-free and sufficiently fast performance was reached. After the subject had been positioned into the scanner, another short practice session followed. The experiment consisted of a total of 252 pseudo-randomized trials, subdivided into 42 blocks. Total scanning time was $45 \mathrm{~min}$.

\section{Behavioral analysis}

For each trial, the RT (the time interval from the cue onset to the release of the home key) was measured. Given that the subject could see the object during the entire trial, but did not know which part to grasp until the LED was switched on, we consider the RT as an index of movement planning time (Fig. $1 D$ ). We also considered the following behavioral measures: movement execution time (MT; the time interval from the release of home key to the removal of the object from its support), transport time (TrT; the time interval from the removal of the object to the insertion of the object into the slot), and return time (the time interval from the insertion of the object into the slot to the return of the hand on the home key). In addition, we recorded whether the object manipulation was correctly performed. Effects of object size (LARGE, SMALL) on RTs and MTs measured during the scanning session were assessed using a paired $t$ test. The behavioral analysis of the kinematic control experiment is described in the supplemental material (available at www.jneurosci.org).

\section{Image acquisition}

Images were acquired using a Siemens (Erlangen, Germany) 3T Trio MRI system, using the body coil for radiofrequency transmission and an eight-channel phased-array surface head coil for signal reception. Blood oxygenation level-dependent (BOLD)-sensitive functional images were acquired using a single-shot gradient echo planar imaging (EPI) sequence [repetition time (TR)/echo time (TE), $2.3 \mathrm{~s} / 40 \mathrm{~ms} ; 31$ transversal slices; voxel size, $3.5 \times 3.5 \times 3.5 \mathrm{~mm}]$. At the end of the scanning session, anatomical images were acquired using an MPRAGE (magnetization prepared rapid gradient echo) sequence (TE/TR, 3.93/2300 ms; $192 \mathrm{sag-}$ ittal slices; voxel size, $1.0 \times 1.0 \times 1.0 \mathrm{~mm}$; field of view, $256 \mathrm{~mm}$ ).

\section{Imaging data analysis}

Preprocessing. Functional data were spatially preprocessed with SPM2 and statistically analyzed with SPM5 (Statistical Parametric Mapping, www.fil.ion.ucl.ac.uk/spm). The first five volumes of each participant's data set were discarded to allow for T1 equilibration. The image time series were spatially realigned using rigid-body transformations and a sinc interpolation algorithm (Friston et al., 1995).

The time series for each voxel was realigned temporally to acquisition of the middle slice. Subsequently, images were normalized onto a custom Montreal Neurological Institute (MNI)-aligned EPI template (based on 24 male brains acquired on the Siemens Trio at the F. C. Donders Centre) using both linear and nonlinear transformations.

Finally, the normalized images were spatially smoothed using an isotropic $10 \mathrm{~mm}$ full-width at half-maximum Gaussian kernel. Each participant's structural image was spatially coregistered to the mean of the functional images (Ashburner and Friston, 1997) and spatially normalized using the same transformation matrix as applied to the functional images.

DCM. Effective connectivity is defined as the influence that one neural system exerts over another (Friston, 1995). DCM aims to estimate and make inferences about the causal influences or coupling among brain regions and how this coupling is modulated by the experimental manipulation (Friston et al., 2003).

The first step is to construct a biologically and anatomically plausible model of interacting cortical regions at the neuronal level (not accessible by fMRI). The dynamic causal model here is an input state-output system, modeled by bilinear differential equations (Friston et al., 2003), in which changes in the states (the neuronal population activities) are modeled on the basis of the known inputs and measured outputs:

$$
\frac{d z}{d t}=\left(A+\sum_{j=1}^{m} u_{j} B^{(j)}\right) z+C u .
$$

Here, $z$ is the state vector (with each state variable representing the population activity of one region in the model), $t$ is continuous time, and $u_{j}$ is the $j$ th input to the modeled system (i.e., some experimentally controlled manipulation). In this state equation, the $A$ matrix contains the "intrinsic" or "fixed" connection strengths between the modeled regions, and the $B^{(1)} \ldots B^{(m)}$ matrices represent the context-dependent modulation of these connections (e.g., by task, as an additive change). The $C$ matrix represents the strengths of direct ("driving") inputs to the modeled system (e.g., sensory stimuli). Note that the inputs $u$ correspond to designed causes (e.g., boxcar or $\delta$ stimulus functions), such as those used to form design matrices in conventional fMRI analyses. The outputs correspond to the observed BOLD time series of the selected volumes of interest (VOIs). Being based on first-order differential equations, the parameters in a DCM denotes the speed or rate of change of neuronal activity (in hertz) in one area as induced by an input or by the output from another area, respectively. The focus of DCM is typically on the $B$ parameters, the modulatory effects. Estimating these parameters and making statistical inference about them enables us to investigate whether grasping objects of different size is associated with changes in coupling among the brain areas in the parieto-frontal circuits under study.

In DCM, the neuronal model described above is supplemented with a hemodynamic forward model of how neuronal activity is transformed into a measured BOLD response. Neuronal and hemodynamic parameters are estimated by Bayesian inversion using an expectation maximization algorithm and a Laplace approximation to the posterior density (for details, see Friston et al., 2003). In brief, the E-step estimates the posterior mean by a gradient ascent on the log posterior, whereas the M-step computes the hyperparameters by minimizing the variational free energy.

We used DCM as implemented in SPM5 (Statistical Parametric Mapping, www.fil.ion.ucl.ac.uk/spm). DCMs were constructed for each single subject separately.

The aim of our DCM analysis was to investigate the changes in interregional couplings associated with grasping either SMALL or LARGE objects. Accordingly, for each single subject, we isolated cortical regions that were activated during prehension movements directed to both SMALL and LARGE objects. We implemented this constraint by considering the movement execution period of correctly performed prehension movements (from movement onset to displacement of either SMALL or 
LARGE objects), distinguishing these two effects from the planning phase of the movement [from cue presentation to movement onset (INPUT)]; from the movements occurring after the first object displacement; and from the few incorrect trials. The gray areas in Figure $1 \mathrm{D}$ show the movement planning (RT) and movement execution (MT) phases considered in the model.

General linear model. The relevant time series for the VOIs were extracted from the fMRI data of each individual subject on the basis of event-related analyses in the context of the general linear model. Singlesubject models consisted of regressors separately describing the movement planning phase (RT) for all visual stimuli (INPUT) and the movement execution phase (MT) for object [split into distinct regressors for grasping movements directed toward the large (LARGE) and the small (SMALL) part of the object]. Trial durations were defined on the basis of the behavioral measurements during the experiment. In addition, we separately modeled the remaining part of the movement (the sum of the $\operatorname{TrT}$ and the return time), the rotation of the device, and the error trials.

Each effect was modeled on a trial-by-trial basis as a concatenation of square-wave functions: for the RT with onsets time locked to the onset of the LED cue and offsets time locked to the release of the hand from the home key; for both MT regressors with the onsets time locked to the release of the hand from the home key and the offsets time locked to taking off the object from the rail. For the remaining part of the movement, the onsets were time locked to the taking-off of the object, and the offsets were time locked to the return of the hand on the home key. The rotation of the device was modeled as an event with zero duration. The error trials had a standard length of $6 \mathrm{~s}$. Each of the six square-wave functions were then convolved with a canonical hemodynamic response function and downsampled at each scan to generate six regressors modeling the main effects described above (Friston et al., 1995).

Head-movement effects were accounted for as described by Friston et al. (1996) by including a Volterra expansion of the six rigid-body motion parameters as nuisance covariates, which consisted of linear and quadratic effects of the six realignment parameters belonging to each volume and also included spin-history effects as linear and quadratic effects of motion parameters in the previous volume, giving a total of 24 regressors (Lund et al., 2005). Three additional regressors, describing intensities in white matter, CSF, and residual compartment (section outside the brain and skull) were added. This was done to account for image intensity shifts attributable to movement of the hand within the main magnetic field of the scanner (Culham et al., 2003; Verhagen et al., 2006).

For the selection of VOIs for the DCM, we used a conjunction analysis (Nichols et al., 2005) in each single subject to isolate commonalities in cerebral activity evoked during the execution phase of grasping both SMALL and LARGE objects, and we masked this effect by the activity evoked during the presentation of the INPUT (for the details of VOI selection, see below).

To assess these commonalities in cerebral activity at the group level, we ran a random-effects analysis (RFX), which served as a guideline in the VOI selection. For this analysis, contrasts of the parameter estimates for SMALL and LARGE were calculated for each single subject and entered into a one-sample $t$ test, respectively. Subsequently, statistical parametric maps (SPMs) of the $t$ statistic for the effects of SMALL and LARGE (Nichols et al., 2005) masked by the visual cues (INPUT) were created, with the degrees of freedom corrected for nonsphericity at each voxel. Inferences were drawn at the voxel level, corrected for multiple comparisons using family-wise error (FWE) correction $(p<0.05)$ across the whole brain. Part of these data have been previously used to address a different issue (Majdandžić et al., 2007).

\section{Anatomical inference}

Anatomical details of significant signal changes were obtained by superimposing the SPMs on the structural images of each subject in MNI coordinates. The atlas of Duvernoy et al. (1991) was used to identify relevant anatomical landmarks. When applicable, Brodmann's areas (BAs) were assigned on the basis of the SPM anatomy toolbox (Eickhoff et al., 2005) (i.e., the anatomical position of our significant clusters and local maxima was tested post hoc against published three-dimensional probabilistic cytoarchitectonic maps). When the literature used for VOI selection reported the stereotaxical coordinates in Talairach space, these coordinates were converted to coordinates in MNI space by a nonlinear transform of Talairach to MNI (http://imaging.mrc-cbu.cam.ac.uk/ imaging/MniTalairach).

\section{Selection of VOI: general procedures}

For each single subject, we selected VOIs corresponding to the putative human equivalents of area V3A, area V6A, the AIP area, PMv, and PMd. Despite the difficulties of defining homologies between macaque and human brain areas (Culham et al., 2006), we chose to use these specific functional labels derived from monkey electrophysiological studies to facilitate the comparison of results across species. However, given that we cannot reliably distinguish hand and arm fields within PMv (i.e., F5 and F4, respectively), or caudal/rostral portions of PMd (i.e., F2 and F7, respectively) (Geyer et al., 2000), we have chosen to use generic functional labels such as PMv and PMd (Wise, 1997).

Given that our right-handed subjects performed the task with their dominant arm, and given the left-hemispheric dominance of several aspects of motor control (de Lange et al., 2006), we restricted our analysis to the left hemisphere.

The subject-specific location of the VOIs was guided both anatomically and functionally: anatomically by the stereotaxical coordinates reported in the literature and known anatomical landmarks (see below for details) and functionally by the group maxima obtained from the random-effects conjunction analysis, if applicable. For each subjectspecific VOI, we considered the first eigenvariate of all suprathreshold voxels (uncorrected, $p<0.05$ ) within a $6 \mathrm{~mm}$ radius around the selected subject-specific maximum. These time series were adjusted for the rotation of the device, error trials, head-movement artifacts, and intensity shifts in the magnetic field attributable to arm movement. The time series were also adjusted for the remaining part of the movement to ensure that we only considered the coupling changes during the preparation and execution phase of the movement (Fig. 1D). Therefore, each VOI time series solely reflects the activity evoked during the period starting with the presentation of the instruction cue and ending with the removal of the object from its slot.

For the parietal and frontal VOIs, the subject-specific VOIs were extracted from the conjunction analyses of the effects evoked by both LARGE and SMALL movements, masked by the activity evoked by the INPUT. For area V3A, which served as the area in our models where the visual inputs entered, each subject-specific VOI was selected on basis of the activity evoked by the visual INPUT alone.

\section{Selection of VOI: anatomical details}

The VOI labeled as V3A was selected using the stereotaxical coordinates reported by (Tootell et al., 1997) as a guideline. However, given the absence of individual retinotopic maps, the V3A VOI should be considered as a general area of major visual input, rather than a precise delineation of V3A.

Area V6 and V6A together form what is called the V6 complex. Pitzalis (2006) reported human V6 to be located in or near the posterior branch of the dorsal end of the parieto-occipital sulcus (POS), centered at -11 , $-77,46$. On the basis of macaque anatomy, it seems reasonable to assume that area V6A would lie more dorsally than V6 along the anterior bank of the POS. Given that V6A is a well established cerebral area, defined on the basis of electrophysiological, anatomical, and hodological criteria (Matelli et al., 1998; Galletti et al., 1999; Luppino et al., 2005), we prefer to refer to this general region as V6A rather than as the parietal reach region (PRR). PRR is known to be crucially involved in reaching (Snyder et al., 2000; Andersen and Buneo, 2002; Buneo et al., 2002), and V6A was suggested to represent a portion of it (Batista et al., 1999), but more recent findings suggest that PRR is located in the medial intraparietal cortex (Calton et al., 2002; Gail and Andersen, 2006). The AIP VOI was selected on the basis of the SPM Anatomy Toolbox (Eickhoff et al., 2005). Additionally, the junction between the postcentral and the intraparietal sulci was used as a landmark on a subject-by-subject basis (Culham et al., 2006).

The ventral premotor (PMv) VOI was selected on the basis of stereotaxical coordinates from neuroimaging studies dealing with precision grasping tasks (Ehrsson et al., 2000; Kuhtz-Buschbeck et al., 2001). 
Table 1. Anatomical connectivity: summary of the available evidence on the basis of tracer studies in macaque monkeys

\begin{tabular}{ll}
\hline Connection & References \\
\hline V3A $\rightarrow$ V6A & Shipp et al., 1998; Galletti et al., 2001 \\
V6A $\rightarrow$ V3A & Shipp et al., 1998; Galletti et al., 2001 \\
V6A $\rightarrow$ PMd & Matelli et al., 1998; Shipp et al., 1998; Caminiti et al., 1999; \\
& Marconi et al., 2001 \\
PMd $\rightarrow$ V6A & Tanne et al., 1995; Caminiti et al., 1999; Marconi et al., 2001; \\
& Luppino et al., 2005 \\
V3A $\rightarrow$ AIP & Nakamura et al., 2001 \\
AIP $\rightarrow$ V3A & Nakamura et al., 2001 \\
AIP $\rightarrow$ PMv & Matelli et al., 1986 \\
PMv $\rightarrow$ AIP & Matelli et al., 1986
\end{tabular}

${ }^{a}$ Regions connected via V6.

${ }^{b}$ Regions connected via LIP (lateral intraparietal sulcus).

The dorsal premotor (PMd) VOI was selected on the basis of stereotaxical coordinates from neuroimaging studies dealing with grasping tasks (Ehrsson et al., 2000). Similar coordinates for both PMv and PMd were used in a transcranial magnetic stimulation (TMS) study (Davare et al., 2006) that confirmed the involvement of these particular precentral regions in visuomotor transformations during the performance of grasping movements.

\section{DCM analysis and inference}

We considered the anatomical model consisting of the VOI as described above with reciprocal connections between them (see Fig. 4, Original model). Table 1 provides the anatomical background for each connection of the model.

The instruction cues (INPUT) that triggered the preparation of the appropriate motor program were fed into V3A. The resulting perturbation was then allowed to propagate throughout the model via interconnections from V3A to V6A, terminating in the PMd, and from V3A to AIP, terminating in PMv. Object size (SMALL, LARGE) served as a modulatory influence on the connections but affected the forward connections only.

The central question was whether the forward connections in the model were differentially modulated by object size, especially whether they were more strongly modulated by grasping a small object (SMALL) compared with a large object (LARGE) during the execution of the movement.

To test whether the modeled processes were expressed consistently across subjects, the subject-specific intrinsic couplings and modulatory effects were entered into separate one-sample $t$ tests (two-sided, statistical threshold, $p<0.05$ ). Second, and most importantly, we tested our hypothesis that grasping a small object changed the coupling between the regions more than grasping a large object, by entering the modulatory parameters of each connection into one-sided paired $t$ tests, testing at the group level whether the modulatory effect of SMALL was larger than the effect of LARGE. The null hypotheses were rejected at a significance level of $p<0.05$.

\section{Model selection}

For any given research question, several alternative hypotheses (with associated models) usually exist. A model selection approach can be used to compare competing models. To test whether our original model was superior to possible alternative models, we applied Bayesian inference to the models themselves (Penny et al., 2004). Bayes factors (i.e., ratios of model evidences) were used to compare different models. Following the classification by Raftery (1995), a decision among models is made if the Bayes factor is at least 3 ("positive evidence"). The model with the highest evidence is a model that is optimally balanced with regard to model fit (accuracy) and model complexity. In the context of DCM, suitable approximations to the model evidence are given by the Bayesian Information Criterion (BIC) and Akaike Information Criterion (AIC) (for details, see Penny et al.,2004). Because BIC is biased toward simpler models and AIC is biased toward more complex models, a model is selected only
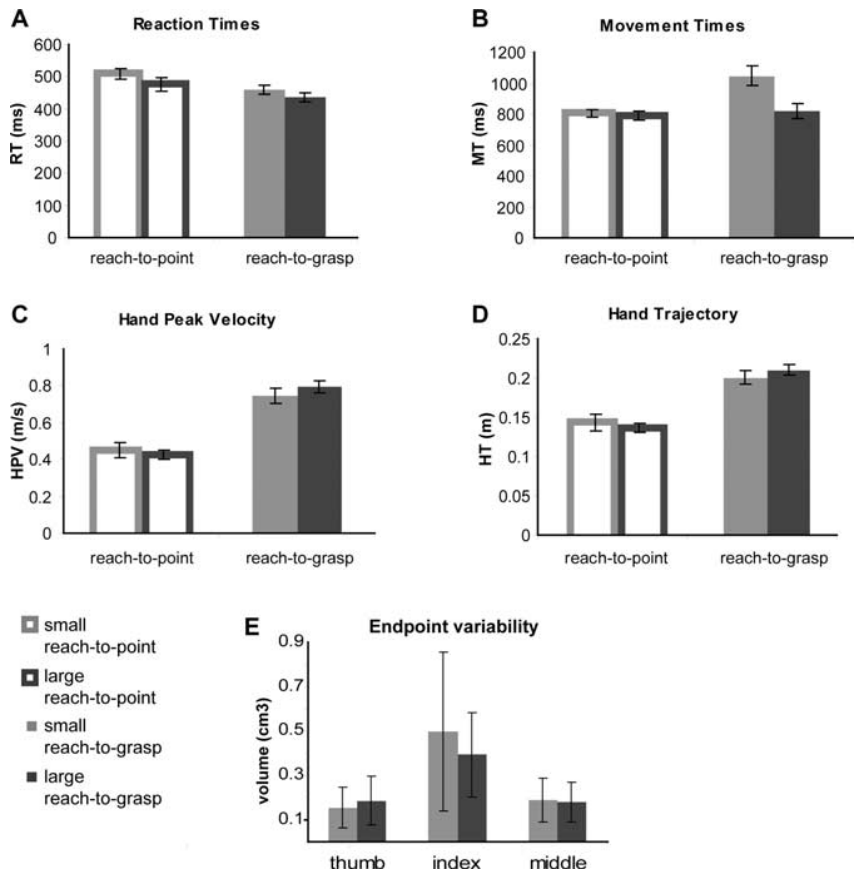

Figure 2. Behavioral results: kinematic control experiment. Movement parameters (A-D) during reaching-to-point (no lining) and reaching-to-grasp (bold lining) trials and the endpoint variability $(\boldsymbol{E})$ during reaching-to-grasp trials toward the small (light gray) and large (dark gray) objects are shown. $\boldsymbol{A}-\boldsymbol{D}$, The histograms illustrate the group means ( \pm SEM) as a function of movement type and object size for the following movement parameters: $A, \mathrm{RT} ; \boldsymbol{B}$, MT; $\boldsymbol{C}$, HPV; $\boldsymbol{D}$, HT. $\boldsymbol{E}$, Histogram illustrates the group mean ( \pm SEM) as a function of object size of the three-dimensional $95 \%$ confidence intervals of the end-point position of the thumb, index finger, and middle finger.

when AIC and BIC concur. The more conservative Bayes factor of the two criteria is then used for the final model selection.

The optimal model may vary across subjects. To decide about the optimal model at the group level, we computed the group Bayes factor (GBF) for each model comparison. Because Bayes factors are independent probability ratios, the GBF can be computed by multiplying the individual Bayes factors of the same model comparison across subjects. However, in case of outliers, GBFs can be delusive (for an example, see Stephan et al., 2007). Therefore, we additionally computed the positive evidence ratio (PER) [i.e., the number of subjects in whom there is positive (or stronger) evidence for model A divided by the number of subjects with positive (or stronger) evidence for model B] (Stephan and Penny, 2007).

\section{Results}

\section{Behavioral performance}

During scanning, the subjects performed the task accurately (average error rate, $1.9 \pm 0.5 \%)$. RTs were shorter when subjects were instructed to grasp the large part of the object [main effect of object: RT, $T_{(1,16)}=3.595 ; p=0.002$; small, $764 \pm 48 \mathrm{~ms}$ (mean \pm SEM); large,729 $\pm 45 \mathrm{~ms}$ (mean \pm SEM)]. The MTs were shorter as well when subjects were instructed to grasp the large part of the object (main effect of object: MT, $T_{(1,16)}=8.002$; $p<0.001$; small, $665 \pm 23 \mathrm{~ms}$ (mean \pm SEM); large, $560 \pm 17 \mathrm{~ms}$ (mean \pm SEM) $]$.

During the kinematic control experiment, subjects' performance was comparable to that measured during the fMRI scanning session. Figure 2 illustrates the movement parameters for the reaching-to-grasp and reaching-to-point trials toward small and large objects. Subjects' RTs (Fig. 2A) and MTs (Fig. 2B) were shorter when they were instructed to grasp the large part of the object than when they grasped the small part of the object (RT, 
main effect of object size: $F_{(1,5)}=9.38, p=0.028$; MT, main effect of object size: $\left.F_{(1,5)}=43.75, p<0.001\right)$. This is a replication of the behavioral findings obtained in the fMRI study. Furthermore, in the control experiment, subjects' RTs were longer when they had to make a reaching-to-point movement than when they had to make a reaching-to-grasp movement (RT, main effect of movement type: $\left.F_{(1,5)}=15.63 ; p=0.011\right)$. This finding indicates that, in this experimental setting, reaching to grasp the large object was not equivalent to reaching to point. Accordingly, post hoc analysis revealed longer RTs preceding reaching-to-point than reachingto-grasp movements toward the large object. We obtained similar results when considering early kinematic parameters such as peak velocity of the hand (HPV) (Fig. 2C): HPV also differed between movement types, with faster movements during reaching-to-point trials (HPV, main effect of movement type: $\left.F_{(1,5)}=94.53 ; p<0.001\right)$. Furthermore, subjects' MTs were sensitive to the size of the object to be grasped, but not to the size of the object to be pointed at (MT, interaction between object size and movement type: $\left.F_{(1,5)}=18.78 ; p=0.007\right)$. In addition, although the MTs of the reaching-to-grasp and reaching-to-point trials were quite similar, on average (MT, main effect of movement type: $\left.F_{(1,5)}=5.56 ; p=0.065\right)$, the hand trajectory $(\mathrm{HT})$ (Fig. 2D) was substantially longer when subjects were reaching to grasp (HT, main effect of movement type: $F_{(1,5)}=54.05 ; p<$ 0.001 ). As seen for the movement time, the HT was also influenced by the size of the object to be grasped, but not by the size of the object to be pointed at (HT, interaction between object size and movement type: $\left.F_{(1,5)}=18.42 ; p=0.008\right)$.

There were also obvious and trivial differences between the finger movements evoked by the reaching-to-grasp condition: the maximal grip aperture (MGA) between thumb and index finger was sensitive to the size of the object to be grasped, with the difference in the opening of the fingers scaled to the difference in the size of the small and large parts of the object. Finally, a post hoc paired $t$ test between reaching to grasp the large and reaching to grasp the small object showed a significant difference in MGA [MGA: effect of object size, $T_{(1,5)}=-17.97, p<0.001$; reachingto-grasp small, $6.4 \pm 0.2 \mathrm{~cm}$ (mean $\pm \mathrm{SEM}$ ); reaching-to-grasp large, $9.8 \pm 0.2 \mathrm{~cm}($ mean \pm SEM) $]$. Together, these results clearly indicate that, in this experiment, the act of grasping the large object does not reduce to a pointing movement.

Figure $2 E$ illustrates the end-point variability for the thumb, index finger, and middle finger measured during the reachingto-grasp movement toward the small and the large object. This variability index was sensitive, being able to discriminate between the larger end-point variability of the index finger and the variability of the other fingers involved in the grasping movement (main effect of finger type: $\left.F_{(2,10)}=5.139 ; p=0.029\right)$. In contrast, the size of the object to be grasped did not influence the end-point variability of the grasping movement (main effect of object size: $\left.F_{(1,5)}=2.788 ; p=0.156\right)$. Therefore, we infer that, in this experimental setting, there are no significant differences in the spatial accuracy demands evoked by reaching-to-grasp movements toward objects of different size.

\section{Imaging data: VOI}

The random-effects conjunction analysis for the effects of SMALL and LARGE (Nichols et al., 2005) masked by the visual cues (INPUT) showed extensive common cerebral activity over occipital, parietal, and frontal regions (FWE corrected, $p<0.05$ ). Figure $3 A$ shows the common cerebral activity within the VOI used by the DCM on a three-dimensional-rendered brain.

On the basis of a priori anatomical information on the puta-
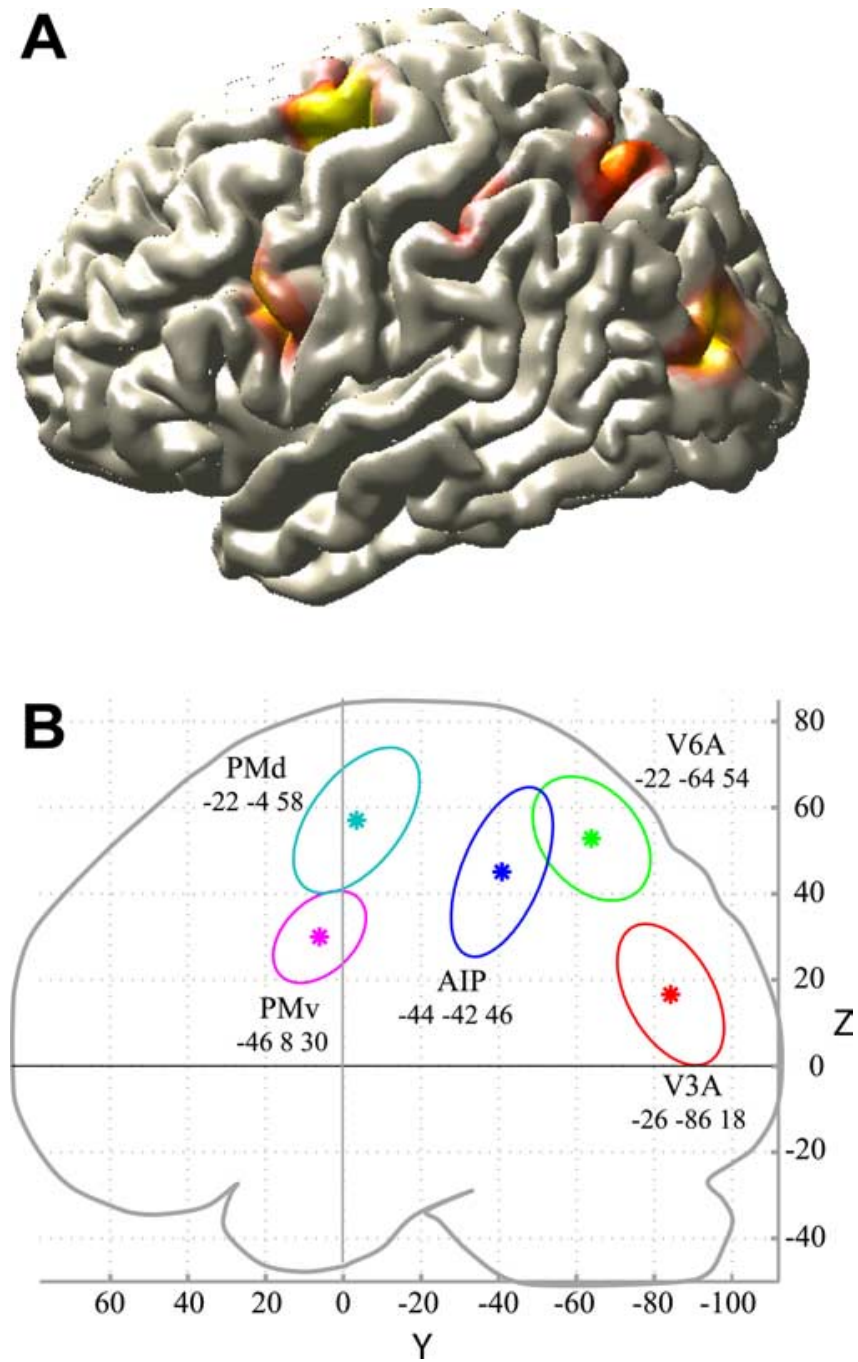

Figure 3. Imaging results. A, RFX. Cerebral activity of the RFX within the VOl used by the DCM on a three-dimensional-rendered brain. The RFX showed extensive common cerebral activity over occipital, parietal, and frontal regions (FWE corrected, $p<0.05$ ). B, Subject-specific VOI. Average group coordinates $(*)$ of the single subject VOls in the $y$ and $z$ directions and variability of the single subject coordinates ( $95 \%$ confidence intervals for each of the five regions) are shown. In the $x$ direction, the average group coordinates for AIP and V6A and for PMd and PMv are separated by 22 and $26 \mathrm{~mm}$, respectively.

tive location of the nodes in our network, we determined subjectspecific VOIs as described in Materials and Methods. For the input region $\mathrm{V} 3 \mathrm{~A}$, regions in the left middle occipital gyrus were selected that responded strongly to the visual INPUT. The average group coordinate $(-26,-86,18)$ was slightly superior to the upper border of V3A as defined by Tootell et al. (1997) and close $(<10 \mathrm{~mm})$ to the stereotaxic coordinates reported by Pitzalis et al. (2006).

The average group coordinates for V6A $(-22,-64,54)$ fell more anteriorly, laterally, and dorsally than the average coordinates for V6, as reported by Pitzalis (2006). This spatial relationship is consistent with the relative position of V6A and V6 in the macaque brain (Galletti et al., 1999).

The AIP activity obtained in the RFX $(-40,-52,48)$ was located within the standard $40 \%$ probabilistic boundary (Eickhoff et al., 2005) of cytoarchitectonically defined hIP2 in the lower bank of the AIP sulcus, an anatomical region suggested to correspond to AIP in the macaque (Choi et al., 2006). The singlesubject coordinates for AIP (average coordinates over subjects: 
Table 2. Effective connectivity

\begin{tabular}{|c|c|c|c|c|c|c|c|c|c|c|c|}
\hline \multirow[b]{2}{*}{ Connections } & \multicolumn{3}{|c|}{ Intrinsic connectivity $^{a}$} & \multicolumn{3}{|c|}{ Modulation of SMALL ${ }^{b}$} & \multicolumn{3}{|c|}{ Modulation of $\mathrm{LARGE}^{b}$} & \multicolumn{2}{|l|}{ SMALL vs LARGE } \\
\hline & Rate constants & SEM & $p$ values & Rate constants & SEM & $p$ values & Rate constants & SEM & $p$ values & Rate constants & $p$ values \\
\hline V3A to V6A & 0.468 & 0.064 & 0.000 & $0.099(21 \%)$ & 0.030 & 0.004 & $0.031(7 \%)$ & 0.011 & $0.015^{d}$ & 0.068 & $0.045^{d}$ \\
\hline V3A to AIP & 0.216 & 0.078 & $0.014^{d}$ & $0.066(31 \%)$ & 0.023 & 0.010 & $0.003(1 \%)$ & 0.021 & 0.895 & 0.064 & $0.032^{d}$ \\
\hline V6A to PMd & 0.421 & 0.068 & 0.000 & $0.019(5 \%)$ & 0.011 & 0.100 & $0.014(3 \%)$ & 0.005 & 0.009 & 0.004 & 0.379 \\
\hline AIP to PMv & 0.251 & 0.093 & $0.016^{d}$ & $0.026(10 \%)$ & 0.008 & 0.007 & $0.007(3 \%)$ & 0.006 & 0.230 & 0.019 & $0.030^{d}$ \\
\hline AIP to V3A & 0.073 & 0.019 & 0.001 & & & & & & & & \\
\hline V6A to V3A & 0.111 & 0.032 & 0.003 & & & & & & & & \\
\hline PMv to AIP & 0.038 & 0.012 & 0.006 & & & & & & & & \\
\hline PMd to V6A & 0.068 & 0.020 & 0.003 & & & & & & & & \\
\hline INPUT to V3A & 0.158 & 0.028 & 0.000 & & & & & & & & \\
\hline
\end{tabular}

${ }^{a}$ Average rate constants [rate of change of neuronal activity (hertz) in one area as induced by another] over subjects for the intrinsic connections and their $p$ values. The intrinsic connectivity refers to the impact that one region exerts over another on the basis of the overall experimental context, rather than in relation to a precise experimental perturbation.

${ }^{b}$ The modulatory effects of object size on the forward connections over subjects (i.e., changes in connection strength). Rate constants are expressed in real values and in percentage change relative to the intrinsic connectivity.

'A contrast (SMALL vs LARGE) testing whether the interregional couplings are more strongly modulated during grasping a SMALL than during grasping a LARGE object.

${ }^{d}$ Significant results that would not survive Bonferroni correction.

$-44,-42,46)$ varied between $y=-32$ [similar to the coordinates reported by Simon et al. (2002)] and $y=-50$ [closer to the coordinates reported by Culham et al. (2003)].

The average group coordinates for PMv $(-46,8,30)$ were located within the $50 \%$ probabilistic boundary of cytoarchitectonically defined BA 44 (Eickhoff et al., 2005) and were close $(<10 \mathrm{~mm})$ to the coordinates reported by Kuhtz-Buschbeck et al. (2001) and Ehrsson et al. (2000) during performance of grasping tasks.

The average group coordinates for PMd $(-22,-4,58)$ were located within the $30 \%$ probabilistic boundary of cytoarchitectonically defined BA6 and slightly medial to the coordinates of the premotor hand area as reported by Ehrsson et al. (2000).

Figure $3 B$ shows the average group coordinates of the VOIs $\left(^{*}\right)$ in the $y$ and $z$ directions and the between-subject variability in 95\% confidence interval ellipsoids.

\section{Effective connectivity}

Table 2 (column 1) summarizes the average rate constants (in hertz) for the intrinsic connections and input over subjects. During preparation and execution of a visually guided grasp, the strength of each intrinsic connection, both forward and backward, within our model was significantly different from zero. Table 2 also shows the average rate constants over subjects for the modulatory effects of executing a grasping movement toward a SMALL (column 4) or LARGE (column 7) object. It is useful to interpret the magnitude of these modulatory changes in relation to the values of the intrinsic connections. For example, the coupling estimate for the connection from V3A to V6A increases from 0.47 to 0.57 when grasping the small object, which corresponds to an increase in coupling strength of $21 \%$ (note that the modulatory effects are additive; see Eq. 1). For completeness, the rate constants in Table 2 are expressed both in real values and in percentage change relative to the intrinsic connectivity. Figure 5 shows the percentage change in connection strength for connections in which the modulatory effect was significantly different from zero. Within the dorsomedial circuit, grasping movements toward both the SMALL (see Fig. 5A, in green) and LARGE (see Fig. $5 B$, in red) objects increased the coupling from V3A to V6A significantly, whereas the connection from V6A to PMd was enhanced significantly during LARGE only (see Table 2 for $p$ values). In contrast, within the dorsolateral circuit, the couplings, from V3A to AIP and from AIP to PMv, were significantly enhanced only when the SMALL object was grasped.

A contrast (SMALL vs LARGE) on each of the bilinear terms, testing for differences between modulatory effects on each connection, showed a significantly stronger modulation of the interregional coupling during SMALL than during LARGE for three of the four connections. First, the forward connection between V3A and V6A was significantly more enhanced by grasping a small object than a large object $\left(T_{(1,16)}=1.8 ; p=0.045\right)$. In addition, the coupling from V3A to AIP was significantly more modulated by SMALL $\left(T_{(1,16)}=1.97 ; p=0.032\right)$; the same was true for the connection from AIP to PMv $\left(T_{(1,16)}=2.03 ; p=0.03\right)$. The coupling between V6A and PMd did not significantly differ between conditions $\left(T_{(1,16)}=0.17 ; p=0.379\right)$.

\section{Model selection}

One might wonder whether the connectivity architecture of our model is optimal and whether alternative models might account better for the data than our model. To address this issue, we formally compared a series of alternative models (Penny et al., 2004) to select the optimal balance between accuracy and complexity of the model.

The alternative models we tested are shown in Figure 4. For instance, it might be argued that the dorsomedial and dorsolateral streams are not anatomically segregated as suggested in the literature. Recent findings in macaques indicate that V6A is interconnected with AIP (Passarelli et al., 2007). We therefore estimated an alternative model (model A1) including reciprocal connections between V6A and AIP and allowed both SMALL and LARGE conditions to modulate the couplings in both directions (model A1). Table 3 shows the GBF and the PER for each alternative model compared with our original model.

The model comparison between model A1 and the original model shows there is strong evidence (Bayes factor $\geq 150$ ) in favor of the original model. The PER for our original model over model 1 was 9:2, which concurred with the GBF in suggesting that the current model accounted better for the data than the alternative model A1.

Furthermore, F2 and F5 (the monkey homologs of PMd and PMv) are mutually interconnected (Matelli et al., 1986; Marconi et al., 2001). We therefore estimated an alternative model including reciprocal connections between PMd and PMv (model A2). We allowed both SMALL and LARGE conditions to modulate the couplings in both directions between PMd and PMv. It has also been suggested that the grasping-related activity observed in V6A could constitute an efference copy of F2 activity (Galletti et al., 1997). Therefore, we tested whether considering a modulation of 


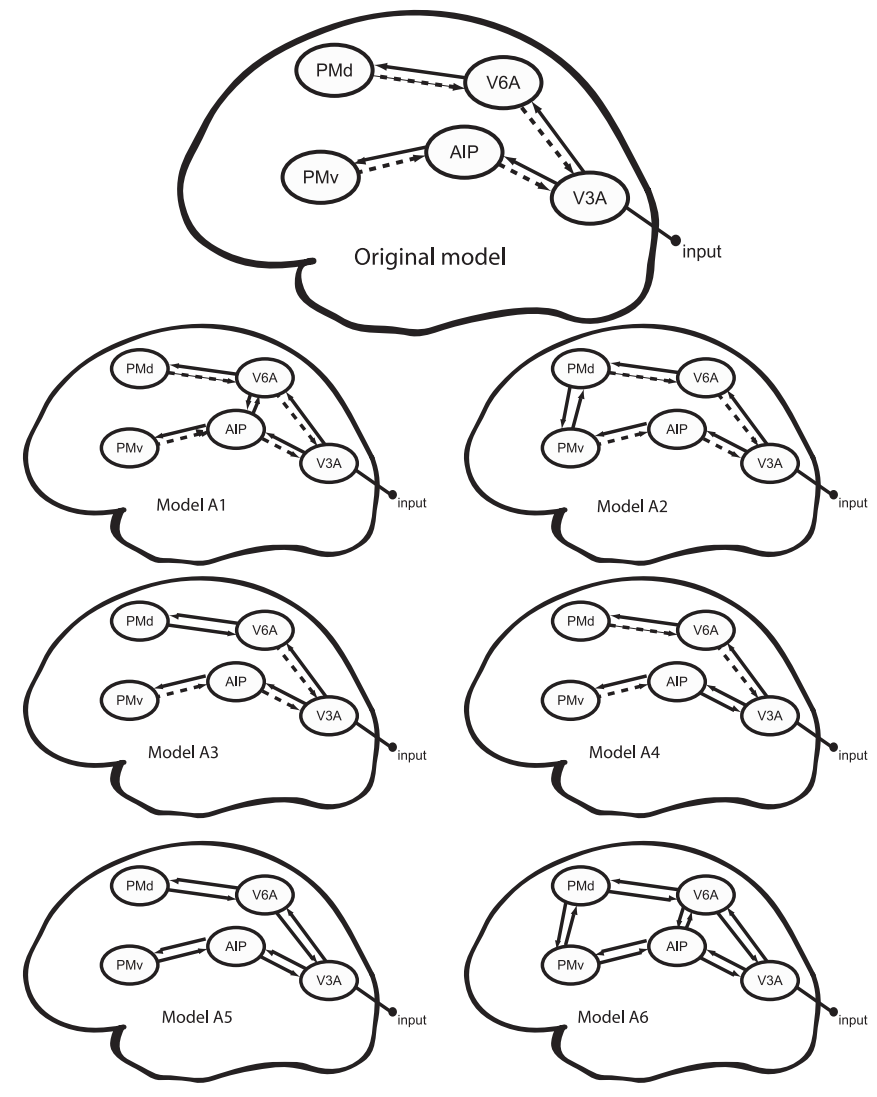

Figure 4. Model comparison. Original and alternative models for the DCM model comparison. Thick black arrows represent connections that are allowed to be modulated by both SMALL and LARGE. Dashed arrows are intrinsic connections not allowed to be modulated. All driving inputs are the instruction cues (INPUT) that triggered the preparation of the appropriate motor program. The INPUT is fed into V3A. The resulting perturbation is allowed to propagate throughout the model via interconnections from V3A to V6A, terminating in the PMd, and from V3A to AIP, terminating in PMv. In the original model, we tested whether the forward connections in this model were differentially modulated by object size (SMALL or LARGE) during the execution of the movement. The alternative models are described in detail in Materials and Methods, Model selection.

object size on the feedback connection from F2 to V6A could improve the model (model A3).

Another way of allowing communication between the streams is through backward connections. In model A4, we tested whether the backward connection between AIP and V3A would be modulated by object size as well. In model A5, we allowed all the backward connections to be modulated by object size. Finally, we wanted to test whether the most complete model (model A6), which included all possible connections and modulations, would be better than our original model. Table 3 summarizes the results from all model comparisons and shows that there is strong evidence (Bayes factor $\geq 150$ ) that the original model is better than any of the alternative models.

\section{Discussion}

We used DCM to evaluate whether and how the inter-regional couplings of an occipito-parieto-frontal network were modulated by grasping objects of different size, while keeping the object position relatively constant. We show that performing prehension movements alters the effective connectivity between occipital, parietal, and frontal regions (Table 2), generating stronger inter-regional couplings during the prehension of smaller objects (Fig. 5). Grasping large objects increased the connectivity of the dorsomedial circuit, whereas grasping small objects increased the connectivity of a mainly dorsolateral circuit, with a degree of overlap between these two circuits (Fig. 5). These findings suggest that the specification of prehension parameters involves different portions of the parieto-frontal network. In the following sections, we elaborate on the implications of these findings for models of the neural control of prehension movements.

\section{Behavioral performance}

The experimental manipulation evoked different types of prehension movements, with different planning and execution phases for the different objects. It might be argued that grasping the large object might have minimized the grasping requirements, making this condition functionally equivalent to a pointing movement. However, the results of directly comparing the kinematics of reaching-to-grasp and reaching-to-point movements toward the large and small objects argue against this possibility. For instance, the MTs were sensitive to the size of the object to be grasped, but not to the size of the object to be pointed at (Fig. 2B). Movement planning times and early kinematics parameters like the HPV were also different during the two types of movements (Fig. 2C). These results indicate that, in this experiment, the act of grasping the large object does not reduce to a pointing movement.

It might be argued that grasping objects of different size could generate differences in movement accuracy. Control measurements of the end-point variability of the thumb, index finger, and middle finger during the reaching-to-grasp movements argue against this possibility (Fig. $2 E$ ).

\section{Connectivity in the dorsolateral circuit (V3A-AIP-PMv)}

Several imaging studies have localized neurovascular responses evoked during visually guided grasping movements, reporting increases in activity from a region located at the junction between the intraparietal and the inferior postcentral sulci [AIP (Toni et al., 2001; Culham et al., 2003)] and from a ventral portion of the precentral gyrus [PMv (Toni et al., 2001)]. Related studies found similar activities during object manipulations (Binkofski et al., 1999; Ehrsson et al., 2000; Johnson-Frey, 2004). Here, we show that there are specific, differential changes in effective connectivity between AIP and PMv during reaching-to-grasp movements. This finding fits with the general notion that the dorsolateral circuit is concerned with controlling grasping parameters of the prehension movement (Jeannerod et al., 1995). In contrast, the couplings between AIP and PMv increased more during the execution of a movement toward a small object than toward a large one. This finding is difficult to reconcile with the notion of dedicated parieto-frontal circuits for reaching and grasping. We suggest that this increased connectivity might reflect the increased on-line control required by grasping small objects. It is known that prehension of objects with small surfaces (relative to finger size) requires a larger degree of visual feedback (Bootsma et al., 1994), and that the kinematic profile of the hand is disproportionally altered when grasping small objects without visual guidance (Chieffi and Gentilucci, 1993). Berthier et al. (1996) also showed that as visual information and object size decreased, subjects had longer movement times, slower speeds, and more asymmetrical hand-speed profiles. We suggest that, during the prehension of small objects, AIP could increase its coupling with $\mathrm{PMv}$ to transform object-centered target representations $(\mathrm{Mu}-$ rata et al., 2000) into motor space (Kurata and Hoshi, 2002) on the basis of incoming visual information of the moving arm (Ochiai et al., 2005). This suggestion follows the notion that the 
Table 3. Model comparison: group Bayes factors (GBF)

\begin{tabular}{lllllll}
\hline & Original vs A1 & Original vs A2 & Original vs A3 & Original vs A4 & Original vs A5 & Original vs A6 \\
\hline GBF & 309.43 & $1.85 \times 10^{18}$ & $2.34 \times 10^{9}$ & $2.26 \times 10^{8}$ & $3.62 \times 10^{19}$ & $1.06 \times 10^{48}$ \\
PER & $9: 2$ & $12: 1$ & $14: 2$ & $14: 2$ & $14: 1$ & $13: 1$ \\
\hline
\end{tabular}

PER, Positive evidence ratio [i.e., the number of subjects in whom there is positive (or stronger) evidence for the original model divided by the number of subjects with positive (or stronger) evidence for the alternative models A1-A6]. All criteria provide strong evidence in favor of the original model.
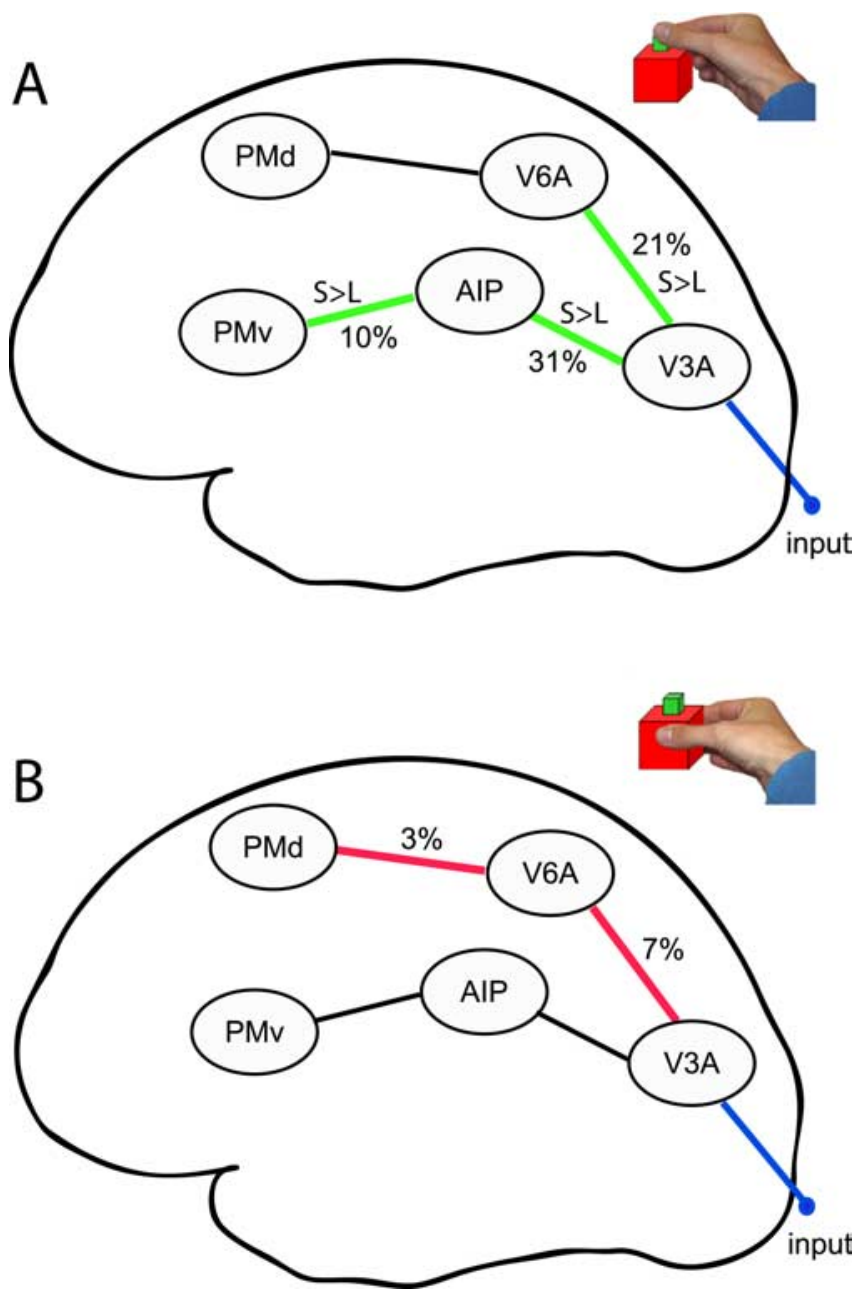

Figure 5. Effective connectivity. Modulatory effects (i.e., significant changes in connection strength) of object size on forward connections in the model (over subjects). $\boldsymbol{A}$, modulatory effects of executing a prehension movement toward a SMALL object (green). $S>L$, The interregional couplings that are significantly stronger modulated during grasping a SMALL than during grasping a LARGE object. $\boldsymbol{B}$, modulatory effects of executing a prehension movement toward a LARGE object (red).

PMv supports a difference vector between the current state of an effector and the target of the movements (Shadmehr and Wise, 2005). The emphasis here is on control, because the modulatory influences of object size on this dorsolateral circuit are related to the execution phase of the prehension movement. This interpretation is in line with recent TMS reports on the crucial role of the AIP region and $\mathrm{PMv}$ during reaching-to-grasp movements ( $\mathrm{Tu}-$ nik et al., 2005; Davare et al., 2006; Rice et al., 2006), in which TMS was only disrupting the movement during the execution phase and not during the planning phase (Rice et al., 2006). It has also been shown that PMv activity reflects dynamical motor parameters specifically during the execution of a movement, and not during its preparation (Xiao et al., 2006).
Connectivity in the dorsomedial circuit (V3A-V6A-PMd)

We found significant increases in effective connectivity between V3A and V6A during movements directed toward both small and large objects and a small but significant change in coupling strength between V6A and PMd during movements toward the large object. The involvement of area V6A in prehension movements is supported by its anatomical connectivity (i.e., direct projections to premotor regions controlling complex proximal and distal arm movements) (Raos et al., 2003). Furthermore, it is known that both V6A and PMd are involved in processing visuospatial information for visual control of arm-reaching movements (Fattori et al., 2001, 2005) and that patients with occipitoparietal lesions show severe impairments in both reaching and grasping (Jeannerod et al., 1994; Milner et al., 2003). Similarly, controlled lesions of V6A in monkeys provoked deficits in reaching, wrist orientation, and grasping (Battaglini et al., 2002). Fattori et al. (2004) recently showed that some V6A cells are specifically modulated during the grasping of visual objects. The increased effective connectivity we observed within the dorsomedial circuit during both types of prehension movements fits with the properties of V6A and with the general notion that the dorsomedial circuit is concerned with specifying arm movements in space (Burnod et al., 1999). In contrast, the coupling between V6A and PMd increased during reaching to grasp toward large objects but not toward small objects (note, however, that the direct comparison between the two conditions did not reveal a significant difference). This finding is not immediately compatible with the notion of a dorsomedial circuit dedicated to reaching movements. We suggest that this increased connectivity might reflect an increased reliance on advance information allowed by grasping large objects. It is known that the PMd supports movement preparation and execution on the basis of advance information (Wise et al., 1997). Under the assumption that the prehension of objects with large surfaces available for finger contact can rely to a larger degree on a prespecified motor plan (Chieffi and Gentilucci, 1993; Berthier et al., 1996), we suggest that the eye-centered, automatic motor plan generated by the superior parietal lobule irrespectively of target characteristics (Pisella et al., 2000, Medendorp et al., 2003) could be forwarded to PMd to incorporate the relative position of target, hand, and eyes (Pesaran et al., 2006) as well as associative rules, if necessary (Toni et al., 2001).

\section{Interpretational limitations}

These results are to be interpreted within the limitations of our modeling approach. Rather than exploring the entire space of model configurations (given our five anatomical VOIs), we used formal model selection procedures (Penny et al., 2004; Stephan and Penny, 2007) to compare models with anatomical plausibility and parsimony. An example of the latter is the choice to feed the external visual input directly into the last common node of the dorsomedial and dorsolateral streams (i.e., V3A), rather than modeling the whole retino-geniculo-striatal pathway. Analogously, we have chosen to avoid modeling primary motor cortex and the cortico-spinal motor output. These simplifications are necessary to make the models computationally tractable and en- 
able us to focus on the most important aspects of the pathways we are interested in, but they may limit the scope of interpretation.

In our second-level analyses, we investigated multiple DCM parameters. Strictly speaking, this necessitates a correction for multiple comparisons. The conventional Bonferroni correction can be too severe a correction when applied to parameter estimates of dynamic system models, because it assumes complete independence between the entities tested. In dynamic system models, however, it is not infrequently the case that conditional dependencies exist among the parameters. Inspection of the posterior covariance matrices of our models revealed low dependencies, thus ensuring good model identifiability. Most of our significant results remained significant after applying Bonferroni correction, including the significant modulation of connections by object size (Table 2). However, the differential effect of modulation by SMALL versus LARGE did not survive correction.

\section{Conclusion}

We have explored the pattern of inter-regional couplings during a reaching-grasping task. Analysis of the changes in effective connectivity in an anatomically grounded occipito-parietofrontal network during task performance revealed that dorsolateral and dorsomedial portions of the parieto-frontal network are modulated differently by prehension movements with different degrees of on-line control. These results argue against a strict dichotomy between the cerebral control of reaching and grasping in humans, as suggested by the two visuomotor channel hypotheses (Jeannerod et al., 1995). We suggest an alternative hypothesis in which the relevance of the dorsolateral and the dorsomedial circuits for prehension is a function of the degree of on-line control required by the movement.

\section{References}

Andersen RA, Buneo CA (2002) Intentional maps in posterior parietal cortex. Annu Rev Neurosci 25:189-220.

Arbib MA (1981) Perceptual structures and distributed motor control. In: Handbook of physiology, Sec 2, The nervous system, Vol II, Motor control, Part 1 (Brooks VB, ed), pp 1449-1480. Bethesda, MD: American Physiological Society.

Ashburner J, Friston K (1997) Multimodal image coregistration and partitioning — a unified framework. NeuroImage 6:209-217.

Batista AP, Buneo CA, Snyder LH, Andersen RA (1999) Reach plans in eyecentered coordinates. Science 285:257-260.

Battaglini PP, Muzur A, Galletti C, Skrap M, Brovelli A, Fattori P (2002) Effects of lesions to area V6A in monkeys. Exp Brain Res 144:419-422.

Berthier NE, Clifton RK, Gullapalli V, McCall DD, Robin DJ (1996) Visual information and object size in the control of reaching. J Mot Behav 28:187-197.

Binkofski F, Buccino G, Posse S, Seitz RJ, Rizzolatti G, Freund H (1999) A fronto-parietal circuit for object manipulation in man: evidence from an fMRI-study. Eur J Neurosci 11:3276-3286.

Bootsma RJ, Marteniuk RG, Mackenzie CL, Zaal FT (1994) The speedaccuracy trade-off in manual prehension-effects of movement amplitude, object size and object width on kinematic characteristics. Exp Brain Res 98:535-541.

Buneo CA, Jarvis MR, Batista AP, Andersen RA (2002) Direct visuomotor transformations for reaching. Nature 416:632-636.

Burnod Y, Baraduc P, Battaglia-Mayer A, Guigon E, Koechlin E, Ferraina S, Lacquaniti F, Caminiti R (1999) Parieto-frontal coding of reaching: an integrated framework. Exp Brain Res 129:325-346.

Calton JL, Dickinson AR, Snyder LH (2002) Non-spatial, motor-specific activation in posterior parietal cortex. Nat Neurosci 5:580-588.

Caminiti R, Genovesio A, Marconi B, Mayer AB, Onorati P, Ferraina S, Mitsuda T, Giannetti S, Squatrito S, Maioli MG, Molinari M (1999) Early coding of reaching: frontal and parietal association connections of parieto-occipital cortex. Eur J Neurosci 11:3339-3345.

Chieffi S, Gentilucci M (1993) Coordination between the transport and the grasp components during prehension movements. Exp Brain Res 94:471-477.

Choi HJ, Zilles K, Mohlberg H, Schleicher A, Fink GR, Armstrong E, Amunts K (2006) Cytoarchitectonic identification and probabilistic mapping of two distinct areas within the anterior ventral bank of the human intraparietal sulcus. J Comp Neurol 495:53-69.

Culham JC, Danckert SL, Desouza JF, Gati JS, Menon RS, Goodale MA (2003) Visually guided grasping produces fMRI activation in dorsal but not ventral stream brain areas. Exp Brain Res 153:180-189.

Culham JC, Cavina-Pratesi C, Singhal A (2006) The role of parietal cortex in visuomotor control: what have we learned from neuroimaging? Neuropsychologia 44:2668-2684.

Davare M, Andres M, Cosnard G, Thonnard JL, Olivier E (2006) Dissociating the role of ventral and dorsal premotor cortex in precision grasping. J Neurosci 26:2260-2268.

de Lange FP, Helmich RC, Toni I (2006) Posture influences motor imagery: an fMRI study. NeuroImage 33:609-617.

Duvernoy HM, Cabanis EA, Vannson JL (1991) The Human brain: surface, three-dimensional sectional anatomy and MRI. Vienna: Springer.

Ehrsson HH, Fagergren A, Jonsson T, Westling G, Johansson RS, Forssberg H (2000) Cortical activity in precision- versus power-grip tasks: an fMRI study. J Neurophysiol 83:528-536.

Eickhoff SB, Stephan KE, Mohlberg H, Grefkes C, Fink GR, Amunts K, Zilles K (2005) A new SPM toolbox for combining probabilistic cytoarchitectonic maps and functional imaging data. NeuroImage 25:1325-1335.

Fattori P, Gamberini M, Kutz DF, Galletti C (2001) “Arm-reaching” neurons in the parietal area V6A of the macaque monkey. Eur J Neurosci 13:2309-2313.

Fattori P, Breveglieri R, Amoroso K, Galletti C (2004) Evidence for both reaching and grasping activity in the medial parieto-occipital cortex of the macaque. Eur J Neurosci 20:2457-2466.

Fattori P, Kutz DF, Breveglieri R, Marzocchi N, Galletti C (2005) Spatial tuning of reaching activity in the medial parieto-occipital cortex (area V6A) of macaque monkey. Eur J Neurosci 22:956-972.

Fogassi L, Gallese V, Buccino G, Craighero L, Fadiga L, Rizzolatti G (2001) Cortical mechanism for the visual guidance of hand grasping movements in the monkey—a reversible inactivation study. Brain 124:571-586.

Friston KJ (1995) Functional and effective connectivity in neuroimaging: a synthesis. Hum Brain Mapp 2:56-78.

Friston KJ, Holmes AP, Worsley KJ, Poline JB, Frith C, Frackowiak RS (1995) Statistical parametric maps in functional imaging: a general linear approach. Hum Brain Mapp 2:189-210.

Friston KJ, Holmes A, Poline JB, Price CJ, Frith CD (1996) Detecting activations in PET and fMRI: levels of inference and power. NeuroImage 4:223-235.

Friston KJ, Harrison L, Penny W (2003) Dynamic causal modelling. NeuroImage 19:1273-1302.

Gail A, Andersen RA (2006) Neural dynamics in monkey parietal reach region reflect context-specific sensorimotor transformations. J Neurosci 26:9376-9384.

Gallese V, Murata A, Kaseda M, Niki N, Sakata H (1994) Deficit of hand preshaping after muscimol injection in monkey parietal cortex. NeuroReport 5:1525-1529.

Galletti C, Fattori P, Kutz DF, Battaglini PP (1997) Arm movement-related neurons in the visual area V6A of the macaque superior parietal lobule. Eur J Neurosci 9:410-413.

Galletti C, Fattori P, Kutz DF, Gamberini M (1999) Brain location and visual topography of cortical area V6A in the macaque monkey. Eur J Neurosci 11:575-582.

Galletti C, Gamberini M, Kutz DF, Fattori P, Luppino G, Matelli M (2001) The cortical connections of area V6: an occipito-parietal network processing visual information. Eur J Neurosci 13:1572-1588.

Galletti C, Kutz DF, Gamberini M, Breveglieri R, Fattori P (2003) Role of the medial parieto-occipital cortex in the control of reaching and grasping movements. Exp Brain Res 153:158-170.

Geyer S, Zilles K, Luppino G, Matelli M (2000) Neurofilament protein distribution in the macaque monkey dorsolateral premotor cortex. Eur J Neurosci 12:1554-1566.

Haggard P, Wing A (1995) Coordinated responses following mechanical perturbation of the arm during prehension. Exp Brain Res 102:483-494.

Jeannerod M (1984) The timing of natural prehension movements. J Mot Behav 16:235-254. 
Jeannerod M (1988) The neural and behavioural organization of goaldirected movements. Oxford: Clarendon.

Jeannerod M, Decety J, Michel F (1994) Impairment of grasping movements following a bilateral posterior parietal lesion. Neuropsychologia 32:369-380.

Jeannerod M, Arbib MA, Rizzolatti G, Sakata H (1995) Grasping objects: the cortical mechanisms of visuomotor transformation. Trends Neurosci 18:314-320.

Johnson-Frey SH (2004) The neural bases of complex tool use in humans. Trends Cogn Sci 8:71-78.

Karnath HO, Perenin MT (2005) Cortical control of visually guided reaching: evidence from patients with optic ataxia. Cereb Cortex 15:1561-1569.

Kuhtz-Buschbeck JP, Ehrsson HH, Forssberg H (2001) Human brain activity in the control of fine static precision grip forces: an fMRI study. Eur J Neurosci 14:382-390.

Kurata K, Hoshi E (2002) Movement-related neuronal activity reflecting the transformation of coordinates in the ventral premotor cortex of monkeys. J Neurophysiol 88:3118-3132.

Lund TE, Norgaard MD, Rostrup E, Rowe JB, Paulson OB (2005) Motion or activity: their role in intra- and inter-subject variation in fMRI. NeuroImage 26:960-964.

Luppino G, Hamed SB, Gamberini M, Matelli M, Galletti C (2005) Occipital (V6) and parietal (V6A) areas in the anterior wall of the parieto-occipital sulcus of the macaque: a cytoarchitectonic study. Eur J Neurosci 21:3056-3076.

Majdandžić J, Grol MJ, van Schie HT, Verhagen L, Toni I, Bekkering H (2007) The role of immediate and final goals in action planning: an fMRI study. Neuroimage 37:589-598.

Marconi B, Genovesio A, Battaglia-Mayer A, Ferraina S, Squatrito S, Molinari M, Lacquaniti F, Caminiti R (2001) Eye-hand coordination during reaching. I. Anatomical relationships between parietal and frontal cortex. Cereb Cortex 11:513-527.

Matelli M, Camarda R, Glickstein M, Rizzolatti G (1986) Afferent and efferent projections of the inferior area 6 in the macaque monkey. J Comp Neurol 251:281-298.

Matelli M, Govoni P, Galletti C, Kutz DF, Luppino G (1998) Superior area 6 afferents from the superior parietal lobule in the macaque monkey. J Comp Neurol 402:327-352.

Medendorp WP, Goltz HC, Vilis T, Crawford JD (2003) Gaze-centered updating of visual space in human parietal cortex. J Neurosci 23:6209-6214.

Milner AD, Dijkerman HC, McIntosh RD, Rossetti Y, Pisella L (2003) Delayed reaching and grasping in patients with optic ataxia. Prog Brain Res 142:225-242.

Murata A, Gallese V, Luppino G, Kaseda M, Sakata H (2000) Selectivity for the shape, size, and orientation of objects for grasping in neurons of monkey parietal area AIP. J Neurophysiol 83:2580-2601.

Nakamura H, Kuroda T, Wakita M, Kusunoki M, Kato A, Mikami A, Sakata H, Itoh K (2001) From three-dimensional space vision to prehensile hand movements: the lateral intraparietal area links the area V3A and the anterior intraparietal area in macaques. J Neurosci 21:8174-8187.

Nichols T, Brett M, Andersson J, Wager T, Poline JB (2005) Valid conjunction inference with the minimum statistic. NeuroImage 25:653-660.

Ochiai T, Mushiake H, Tanji J (2005) Involvement of the ventral premotor cortex in controlling image motion of the hand during performance of a target-capturing task. Cereb Cortex 15:929-937.

Passarelli L, Gamberini M, Baldinotti I, Fattori P, Luppino G, Matelli M, Galletti C (2007) Cortico-cortical connections of dorsal area V6A in the macaque monkey. Paper presented at International Brain Research Organization, World Congress of Neuroscience, Melbourne, Australia, July.

Penny WD, Stephan KE, Mechelli A, Friston KJ (2004) Comparing dynamic causal models. NeuroImage 22:1157-1172.

Pesaran B, Nelson MJ, Andersen RA (2006) Dorsal premotor neurons encode the relative position of the hand, eye, and goal during reach planning. Neuron 51:125-134.

Pisella L, Grea H, Tilikete C, Vighetto A, Desmurget M, Rode G, Boisson D, Rossetti Y (2000) An "automatic pilot" for the hand in human posterior parietal cortex: toward reinterpreting optic ataxia. Nat Neurosci 3:729-736.

Pisella L, Binkofski F, Lasek K, Toni I, Rossetti Y (2006) No doubledissociation between optic ataxia and visual agnosia: multiple substreams for multiple visuo-manual integrations. Neuropsychologia 44:2734-2748

Pitzalis S, Galletti C, Huang RS, Patria F, Committeri G, Galati G, Fattori P, Sereno MI (2006) Wide-field retinotopy defines human cortical visual area V6. J Neurosci 26:7962-7973.

Raftery AE (1995) Bayesian model selection in social research. In: Sociological methodology (Marsden PV, ed), pp 111-163. Cambridge, MA: Blackwell.

Raos V, Franchi G, Gallese V, Fogassi L (2003) Somatotopic organization of the lateral part of area F2 (dorsal premotor cortex) of the macaque monkey. J Neurophysiol 89:1503-1518.

Rice NJ, Tunik E, Grafton ST (2006) The anterior intraparietal sulcus mediates grasp execution, independent of requirement to update: new insights from transcranial magnetic stimulation. J Neurosci 26:8176-8182.

Shadmehr R, Wise SP (2005) Computational neurobiology of reaching and pointing: a foundation for motor learning. Cambridge, MA: MIT.

Shipp S, Blanton M, Zeki S (1998) A visuo-somatomotor pathway through superior parietal cortex in the macaque monkey: cortical connections of areas V6 and V6A. Eur J Neurosci 10:3171-3193.

Simon O, Mangin JF, Cohen L, Le Bihan D, Dehaene S (2002) Topographical layout of hand, eye, calculation, and language-related areas in the human parietal lobe. Neuron 33:475-487.

Smeets JB, Brenner E (1999) A new view on grasping. Motor Control 3:237-271.

Snyder LH, Batista AP, Andersen RA (2000) Intention-related activity in the posterior parietal cortex: a review. Vision Res 40:1433-1441.

Stephan KE, Penny W (2007) Dynamic causal models and Bayesian selection. In: Statistical parametric mapping: the analysis of functional imaging data (Friston KJ, ed), pp 577-585. Amsterdam: Elsevier.

Stephan KE, Marshall JC, Penny WD, Friston KJ, Fink GR (2007) Interhemispheric integration of visual processing during task-driven lateralization. J Neurosci 27:3512-3522.

Tanne J, Boussaoud D, Boyer-Zeller N, Rouiller EM (1995) Direct visual pathways for reaching movements in the macaque monkey. NeuroReport 7:267-272.

Tanne-Gariepy J, Rouiller EM, Boussaoud D (2002) Parietal inputs to dorsal versus ventral premotor areas in the macaque monkey: evidence for largely segregated visuomotor pathways. Exp Brain Res 145:91-103.

Toni I, Rushworth MF, Passingham RE (2001) Neural correlates of visuomotor associations: spatial rules compared with arbitrary rules. Exp Brain Res 141:359-369.

Tootell RBH, Mendola JD, Hadjikhani NK, Ledden PJ, Liu AK, Reppas JB, Sereno MI, Dale AM (1997) Functional analysis of V3A and related areas in human visual cortex. J Neurosci 17:7060-7078.

Tunik E, Frey SH, Grafton ST (2005) Virtual lesions of the anterior intraparietal area disrupt goal-dependent on-line adjustments of grasp. Nat Neurosci 8:505-511.

Ulloa A, Bullock D (2003) A neural network simulating human reach-grasp coordination by continuous updating of vector positioning commands. Neural Netw 16:1141-1160.

Verhagen L, Grol MJ, Dijkerman C, Toni I (2006) Studying visually-guided reach-to-grasp movements in an MR-environment. Paper presented at the 12th Annual Meeting of the Organization of Human Brain Mapping, Florence, Italy, June.

Wise SP, Boussaoud D, Johnson PB, Caminiti R (1997) Premotor and parietal cortex: corticocortical connectivity and combinatorial computations. Annu Rev Neurosci 20:25-42.

Xiao J, Padoa-Schioppa C, Bizzi E (2006) Neuronal correlates of movement dynamics in the dorsal and ventral premotor area in the monkey. Exp Brain Res 168:106-119.

Zaal FT, Bootsma RJ, van Wieringen PCW (1998) Coordination in prehension-information-based coupling of reaching and grasping. Experimental Brain Res 119:427-435. 